\title{
Sun Safe Mode Controller Design for LADEE
}

\author{
Jesse C. Fusco, * Sean S.M. Swei ${ }^{\dagger}$ \\ and Robert H. Nakamura $\ddagger$ \\ NASA's Ames Research Center, Moffett Field, CA 94035-1000
}

\begin{abstract}
This paper presents the development of sun safe controllers which are designed to keep the spacecraft power positive and thermally balanced in the event an anomaly is detected. Employed by NASA's Lunar Atmosphere and Dust Environment Explorer (LADEE), the controllers utilize the measured sun vector and the spacecraft body rates for feedback control. To improve the accuracy of sun vector estimation, the least square minimization approach is applied to process the sensor data, which is proven to be effective and accurate. To validate the controllers, the LADEE spacecraft model engaging the sun safe mode was first simulated and then compared with the actual LADEE orbital flight data. The results demonstrated the applicability of the proposed sun safe controllers.
\end{abstract}

\section{Introduction}

The Lunar Atmosphere and Dust Environment Explorer (LADEE) was a NASA robotic science mission designed to study the lunar atmosphere and dust environment from a low lunar orbit. LADEE, headed by NASA Ames Research Center in partner with NASA Goddard Space Flight Center, was launched on September 7, 2013 UTC, and ended its mission on April 18, 2014. Designed as a small and relatively low cost mission, LADEE carried four instrument payloads: a neutral mass spectrometer, an ultraviolet spectrometer, a dust collector, and a lunar laser communication technology demonstration. ${ }^{1}$

An essential part of a spacecraft's attitude determination and control systems (ADCS) is a simple and reliable sun safe controller that the ADCS can fall back on if the spacecraft experiences any problems. Many safe mode control systems for larger missions, such as those implemented on the Hubble Space Telescope ${ }^{2}$ and the Solar Dynamics Observatory (SDO) ${ }^{3}$ feature a fully redundant set of sensors. However, since LADEE was a low budget enhanced class D mission, complete redundancy was neither required nor affordable. Therefore, it was critical that LADEE's sun safe mode be robustly designed and utilize reliable sensors and actuators. Similar to the hardware used in the SDO sun acquisition mode, ${ }^{4}$ the LADEE ADCS hardware included: Twelve coarse sun sensors, four single axis MEMS rate gyros, and a set of four reaction wheels. Additional sensors were included on the spacecraft for finer attitude control, however they were not required to be included in the sun safe control design.

The LADEE sun safe mode was required to ensure that the spacecraft remained power positive, thermally safe, and payload instrument safe. To accomplish these goals, three different states of sun safe situation were considered: initial (instrument) safing state, controlled roll-yaw state, and eclipse state. To design an effective controller for each sun safe state, we must take consideration of the following specifications. First, the LADEE spacecraft has solar arrays wrapped octagonally about the $Z$-axis, meaning it is nearly axisymmetric about the $X$ - and $Y$-axis, with the peak power position being a cone at a $10^{\circ}$ inclination off of the $X Y$-plane. Second, in order to meet the thermal safety requirement, the spacecraft must spin about the body $Z$-axis at a specified rate, thereby illuminating all faces of the spacecraft with equal amounts of sunlight. Finally, an effective design would mitigate spacecraft momentum accumulation due to the disturbance effect from the lunar gravity gradient. Therefore, the LADEE sun safe controller was designed to address these requirements.

\footnotetext{
*Aerospace Engineer, Engineering Systems Division.

${ }^{\dagger}$ Research Scientist, Intelligent Systems Division, Member.

¥Aerospace Engineer, Emergent Space Technologies, Inc.
} 
To protect a sun sensitive instrument upon entering safe mode, the initial safing state is to maneuver the spacecraft so that the instrument is immediately turned away from the sun. After achieving this, the spacecraft would then transition into the controlled roll-yaw state by slowly spinning about $Z$-axis for thermal regulation while also rotating about the sun vector for momentum management. Figure 1 depicts this controlled roll-yaw motion. The rotation about the sun vector would minimize the total momentum build-up in lunar orbit by allowing the external torques to act in opposite directions on the spacecraft over time. Finally, the eclipse state would be engaged if the sun was blocked by another body, with the objective of minimizing the control effort by not holding any specific attitude against external torques.

Except during the eclipse state, measurements from sun sensors are crucial throughout the safe mode. In this paper, a process which utilizes the least square minimization technique is adopted in determining the sun vector from the sensor measurements. This approach is proven to be effective and accurate. The reaction wheels used for the attitude maneuvers are light weight, yet generate sufficient torque for attitude control. They are placed in a four-sided pyramid configuration, which allows varying torques to be distributed among body axes. The spacecraft spin rates are measured through MEMS rate gyros, which are embedded in each reaction wheel and measuring rates along the wheel's spin axis. A process is presented to convert measured rates into spacecraft inertial spin rates.

The spacecraft attitude determination and control problem has been studied quite extensively in the past, especially with quaternion feedback, see for example,,$^{5,6}$ and the references therein. However, the controllers presented in these works tend to be either model dependent or utilizing scalar gains, which may not be desirable in actual implementation. The mass and inertia properties of LADEE were such that different control gains for each body axis were required. In this paper, the stabilizing controllers are outlined for LADEE's sun safe states, and they are not quaternion based. Instead, a sequential feedback loop closure approach is presented, in which a measured sun vector relative to the spacecraft body-fixed frame is used as the outer-loop "attitude" feedback, in addition to the usual inner-loop rate feedback. It is worth noting that LADEE's safe mode controllers do not intend to control the complete spacecraft attitude, as its attitude about the sun vector is unknown. Such designs are sometimes referred to as a "two-and-a-half-degree-offreedom" controllers. ${ }^{4}$

This paper is organized as follows. In Section II, the background and implementation of coarse sun sensors are discussed, and the least square estimation of the sun vector is presented. Section III describes the reaction wheel dynamics, and Section IV discusses the MEMS rate sensors and presents the process to derive spacecraft spin rates. Section V contains the derivation of overall spacecraft system dynamics, and for these dynamics a set of sun safe mode controllers are outlined in Section VI. Section VII contains simulation results, and Section VIII presents the LADEE flight data that validates the design. Finally, the paper ends with some conclusions.

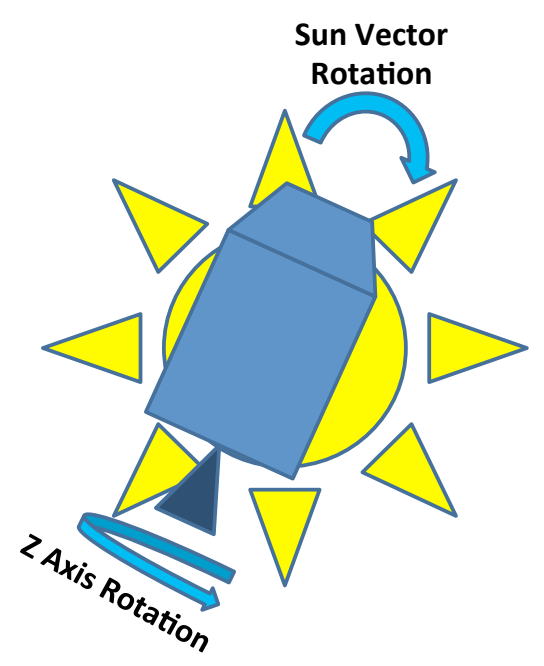

Figure 1. Controlled Roll-Yaw Motion in view of Sun 


\section{Coarse Sun Sensors}

Sun sensors are most critical in LADEE's safe mode control design as the spacecraft's "attitude" is tracked relative to the sun vector $S$. A coarse sun sensor (CSS) is basically a photo-diode that returns a current $I_{\text {out }}$ in proportion to the cosine of the angle between the sensor bore sight and the sun vector $S$, which is approximated by the equation

$$
I_{\text {out }}=K \cos (\phi),
$$

found in the CSS datasheet, where $\phi$ is the angle between the sensor's bore sight and the sun vector, $K$ is the peak current when pointed directly at the sun, i.e. $\phi=0$. Therefore, one sensor does not provide an actual sun vector but rather a cone that the sun vector resides in. As each sensor has a limited field of view (FOV), in order to compute $S$ accurately, the sun must be within the FOV of at least three sensors. Therefore, an array of multiple sun sensors is required to determine the sun vector in any spacecraft orientation.

The LADEE design utilized 12 coarse sun sensors. Several CSS array arrangements were studied and evaluated based on how well they provide coverage over the entire spacecraft FOV, particularly when the spacecraft is oriented perpendicular to the sun vector. The calibration data supplied by manufacturer provide the value of $K$ for each sensor, and by use of the cosine model of Eq. (1) the characterization of each sun sensor can be obtained. Therefore, the first step in processing the $i$ th CSS data is to determine $\phi_{i}$ by solving Eq. (1) and we obtain

$$
\phi_{i}=\cos ^{-1}\left(I_{\text {out }, i} / K_{i}\right), i=1,2, \ldots, 12 .
$$

Next, let the unit sun vector $S$ and the $i$ th CSS bore sight unit vector $B_{i}$ be defined as

$$
S=\left[\begin{array}{c}
s_{x} \\
s_{y} \\
s_{z}
\end{array}\right], B_{i}=\left[\begin{array}{c}
b_{x, i} \\
b_{y, i} \\
b_{z, i}
\end{array}\right]
$$

Then, $\cos \phi_{i}$ can be considered as the inner product of the $i$ th CSS bore sight vector with the sun vector, that is,

$$
\cos \phi_{i}=B_{i}^{T} S=\left[\begin{array}{lll}
b_{x, i} & b_{y, i} & b_{z, i}
\end{array}\right]\left[\begin{array}{c}
s_{x} \\
s_{y} \\
s_{z}
\end{array}\right], i=1,2, \ldots, 12 .
$$

Now, determining the sun vector $S$ from (3) is equivalent to solving the following standard least square minimization problem,

$$
\min \{\|B S-\mu\|: \text { for all }\|S\|=1\},
$$

where $\|\cdot\|$ denotes the vector norm, and

$$
B=\left[\begin{array}{c}
B_{1}^{T} \\
B_{2}^{T} \\
\ldots \\
B_{i}^{T}
\end{array}\right], \mu=\left[\begin{array}{c}
\cos \phi_{1} \\
\cos \phi_{2} \\
\cdots \\
\cos \phi_{i}
\end{array}\right] .
$$

The unique solution to (4) is given by

$$
S=\left(B^{T} B\right)^{-1} B^{T} \mu .
$$

Several variants of the least square problems were experimented. For instance, the sun vector could be derived by using only the three brightest CSS readouts, or by using only those CSS readouts that were above a certain threshold. These results indicated that the sun vector estimation error was large, especially at higher $\phi$ angle. In comparison, the proposed minimum least square approach (4) was found to produce a reasonably accurate sun vector estimate over the entire attitude sphere.

\section{Reaction Wheels}

Reaction wheels are commonly used for spacecraft attitude control. They are essentially a flywheel attached to a brush-less DC motor. As the motor accelerates the flywheel, an equal and opposite torque is 
applied to the spacecraft. Reaction wheels generally produce relatively small torques and are very controllable, thus making them the ideal choice when fine pointing resolution is required.

The LADEE spacecraft design uses four reaction wheels arranged in a four-sided pyramid configuration with base angle $\beta$; as depicted in Figure 2, thus providing one redundancy. The base angle is chosen so that the resultant reaction wheel torques are mostly along the spacecraft's $Z$-axis (where primary rotation will occur), while providing sufficient torque control authority in cross axes.

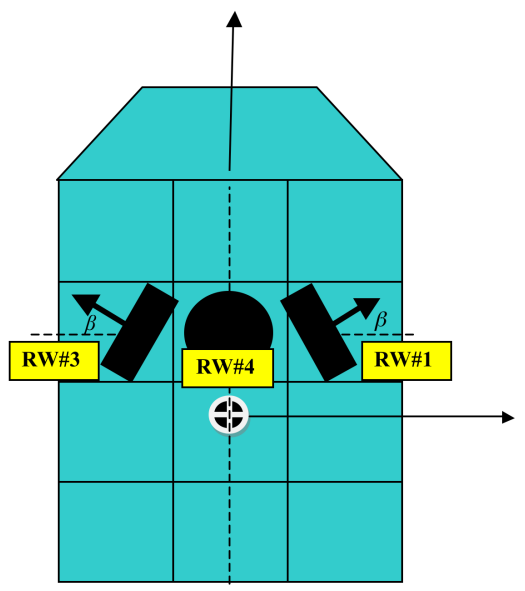

Figure 2. Reaction wheel configuration in LADEE

Let $T$ be a $3 \times 4$ matrix that converts the axial reaction wheel vectors to the body fixed frame,

$$
T=\left[\begin{array}{cccc}
\cos \beta & 0 & -\cos \beta & 0 \\
0 & \cos \beta & 0 & -\cos \beta \\
\sin \beta & \sin \beta & \sin \beta & \sin \beta
\end{array}\right]=\left[\begin{array}{llll}
t_{1} & t_{2} & t_{3} & t_{4}
\end{array}\right],
$$

where $t_{i}$ represents the axial unit vector of the $i$ th wheel about the body fixed frame. Therefore, the total reaction wheel angular momentum relative to the inertial frame, denoted as ${ }^{I} H^{r w}$, is given by

$$
{ }^{I} H^{r w}=T I_{r w} T^{T I} \omega^{b}+T I_{r w}{ }^{b} \omega^{r w},
$$

where ${ }^{I} \omega^{b}$ is the angular velocity of spacecraft relative to the inertial frame, $T$ the conversion matrix given in (6), $I_{r w}=\operatorname{diag}\left\{I_{1}, I_{2}, I_{3}, I_{4}\right\} ; I_{i}$ denotes the $i$ th wheel axial moment of inertia, and ${ }^{b} \omega^{r w}=$ $\left[\Omega_{1}, \Omega_{2}, \Omega_{3}, \Omega_{4}\right]^{T} ; \Omega_{i}$ denotes the $i$ th wheel spin rate relative to the body fixed frame.

\section{MEMS Rate Sensors}

There are several prominent MEMS technologies for measuring angular rate, but almost all of them work by inducing a vibration mode along one axis, then measuring the amplitude of the vibration that is induced around the measurement axis by the Coriolis force. The rate sensors utilized in LADEE's safe mode were of the quartz tuning fork variety. ${ }^{7}$ The four sensors were embedded inside each reaction wheel and aligned with its axial direction, therefore each sensor gave rate data about the reaction wheel spin axis.

Since the MEMS sensors were arranged in the same pyramid configuration as the reaction wheels, the measured rate was overdetermined and therefore processed the same way the CSS data was processed. The spacecraft rotational rates were then extracted as follows,

$$
\underbrace{\left[\begin{array}{c}
\omega_{m_{1}} \\
\omega_{m_{2}} \\
\omega_{m_{3}} \\
\omega_{m_{4}}
\end{array}\right]}_{I \omega^{m}}=\underbrace{\left[\begin{array}{ccc}
\cos \beta & 0 & \sin \beta \\
0 & \cos \beta & \sin \beta \\
-\cos \beta & 0 & \sin \beta \\
0 & -\cos \beta & \sin \beta
\end{array}\right]}_{T^{T}} \underbrace{\left[\begin{array}{c}
\omega_{x} \\
\omega_{y} \\
\omega_{z}
\end{array}\right]}_{I \omega^{b}},
$$


where $\omega_{m_{i}}$ is the measured spacecraft rotational rate at $i$ th wheel. Therefore, the spin rate ${ }^{I} \omega^{b}$ can be obtained by performing the pseudo-inverse to the above and is given by

$$
{ }^{I} \omega^{b}=\left(T T^{T}\right)^{-1} T^{I} \omega^{m}
$$

The pyramid configuration also offers redundancy, allowing failure of one MEMS sensor. As long as the other sensors are functioning properly, three-axis rate measurement can still be derived. However, in this case, the spacecraft spin rate is derived from

$$
{ }^{I} \omega^{b}=\bar{T}\left(\bar{T}^{T} \bar{T}\right)^{-1 I} \omega^{m},
$$

where $\bar{T}^{T}$ is the sub-matrix formed by the rows of $T^{T}$ in (8) that correspond to the functioning sensors. It should be noted that, in actual implementation, the LADEE MEMS rate measurement $\omega_{m_{i}}$ was filtered through a low-pass filter before it was fed back for control design.

\section{Spacecraft System Dynamics}

The angular momentum of the rigid body spacecraft in the inertial frame is given by

$$
{ }^{I} H^{s p}=J^{I} \omega^{b},
$$

where $J$ is the mass moment of inertia of the spacecraft, excluding the reaction wheels. Therefore, the total angular momentum for the spacecraft system is the summation of the reaction wheel angular momentum given in Eq. (7) and the spacecraft angular momentum given in Eq. (10), and it can be described by ${ }^{8}$

$$
\begin{aligned}
{ }^{I} H^{s s} & ={ }^{I} H^{s p}+{ }^{I} H^{r w} \\
& =J^{I} \omega^{b}+T I_{r w} T^{T I} \omega^{b}+T I_{r w}{ }^{b} \omega^{r w} \\
& =J_{a}{ }^{I} \omega^{b}+T I_{r w}{ }^{b} \omega^{r w}
\end{aligned}
$$

where $J_{a}=J+T I_{r w} T^{T}$. Note that in general the reaction wheel inertia is negligible compared to the spacecraft inertia, so in practice $J_{a} \approx J$.

Let $\tau_{\text {ext }}$ denote the total external disturbance torque, and by setting the time derivative of Eq. (11) equal to the external disturbance torque, we obtain

$$
\begin{aligned}
\tau_{e x t} & =\frac{d^{I} H^{s s}}{d t} \\
& =\frac{d^{I} H^{s s}}{d t}+{ }^{I} \omega^{b} \times{ }^{I} H^{s s}
\end{aligned}
$$

Substituting Eq. (11) into above yields,

$$
\begin{aligned}
\tau_{\text {ext }} & =\left(J_{a}{ }^{I} \dot{\omega}^{b}+T I_{r w}{ }^{b} \dot{\omega}^{r w}\right)+{ }^{I} \omega^{b} \times\left(J_{a}{ }^{I} \omega^{b}+T I_{r w}{ }^{b} \omega^{r w}\right) \\
& =\underbrace{\left(J_{a}{ }^{I} \dot{\omega}^{b}+{ }^{I} \omega^{b} \times J_{a}{ }^{I} \omega^{b}\right)}_{\text {Spacecraft }}+\underbrace{\left(T I_{r w}{ }^{b} \dot{\omega}^{r w}+{ }^{I} \omega^{b} \times T I_{r w}{ }^{b} \omega^{r w}\right)}_{\text {Reaction Wheels }} .
\end{aligned}
$$

Let $u=\left[\begin{array}{lll}\tau_{x} & \tau_{y} & \tau_{z}\end{array}\right]^{t}$ be the internal control torque generated by the reaction wheels, then the equal and opposite torque will be applied to the spacecraft. Therefore, Eq. (13) can be rewritten as follows,

$$
J_{a}^{I} \dot{\omega}^{b}=J_{a}^{I} \omega^{b} \times{ }^{I} \omega^{b}+u+\tau_{e x t}
$$

where the control torque $u$ is defined by

$$
\begin{aligned}
u & =-\left(T I_{r w}{ }^{b} \dot{\omega}^{r w}+{ }^{I} \omega^{b} \times T I_{r w}{ }^{b} \omega^{r w}\right) \\
& =-\left(T \tau_{r w}+{ }^{I} \omega^{b} \times T h_{r w}\right)
\end{aligned}
$$

where $\tau_{r w}$ and $h_{r w}$ denote respectively the reaction wheel torque and angular momentum along its axial direction. Eq. (14) represents the dynamics of the spacecraft subject to the control and disturbance inputs. This equation along with the sun vector dynamics, which will be discussed in the sequel, form a complete 
equation of motion for spacecraft attitude control for the sun safe mode. The control torque $u$ can be designed by following many available references, and once it is determined, we can solve Eq. (15) for both $\tau_{r w}$ and $h_{r w}$, which then become the commanded input to the reaction wheels. In Section VI, we will discuss the development of sun safe mode controllers for various scenarios.

As defined earlier, let $S$ be the measured sun vector relative to the body fixed frame. Then, the time derivative of $S$ relative to the inertial frame, denoted as $\frac{{ }^{I} d S}{d t}$, can be described by

$$
\frac{{ }^{I} d S}{d t}=\frac{{ }^{b} d S}{d t}+{ }^{I} \omega^{b} \times S,
$$

where $\frac{{ }^{b} d S}{d t}$ denotes the time rate change of the sun vector $S$ relative to the body fixed frame. Since the sun vector stays almost stationary in the inertial frame, we deduce that $\frac{{ }^{I} d S}{d t} \approx 0$. Therefore, Eq. (16) can be simplified to

$$
\frac{{ }^{b} d S}{d t}=-{ }^{I} \omega^{b} \times S,
$$

which describes the sun vector dynamics relative to the body fixed frame. Note that the sun vector $S$ is determined by processing the on-board sun sensor data as discussed in Section II, and the spacecraft rotational rate ${ }^{I} \omega^{b}$ is obtained by processing the MEMS rate data as given in Eq. (9). As mentioned earlier, Eqs. (14) and (17) together completely describe the spacecraft attitude dynamics, and they are given as follows,

$$
\Sigma:\left\{\begin{aligned}
J_{a} \dot{\omega} & =J_{a} \omega \times \omega+u \\
\dot{S} & =-\omega \times S
\end{aligned}\right.
$$

where, for brevity, we have used $\omega$ to represent the spacecraft spin rate ${ }^{I} \omega^{b}$ and omitted the external disturbance term $\tau_{\text {ext }}$.

\section{Design of Safe Mode Controllers}

The proposed sun safe mode control concept consists of three states: Initial safing, controlled roll-yaw, and eclipse. The detailed development of safe mode controllers and the proof for global asymptotic stability for the spacecraft system (18) can be found in Swei et al. ${ }^{9}$

\section{A. Initial safing state}

The goal of the initial safing state is to ensure that any sun sensitive instrument $p$ is protected from pointing directly at the sun and additionally, that the spacecraft attitude is oriented for peak power generation. Initially, let the sun sensitive instrument's bore sight be pointed along the unit vector of $\hat{B}_{p}$. Upon entering the initial safing state, the spacecraft is commanded such that the sun vector $S$ turns to $-\hat{B}_{p}$. This will ensure that the spacecraft always turns the sun vector away from the instrument $p$. Once the sun vector $S$ is positioned within a certain tolerance of $-\hat{B}_{p}$, the safe mode will then transition into the controlled roll-yaw state. This design assumes that $\hat{B}_{p}$ does not lie perpendicular to the Z-axis, and thus the desired roll-yaw about the sun vector will not violate any constraints. The initial safing controller is essentially a PD controller consisting of an inner rate loop which is commanded by an outer "attitude" loop, where the attitude error $S_{e}$ is calculated as the cross product of the target sun vector $S_{d}$ and the measured sun vector $S$, that is

$$
S_{e}=S \times S_{d}
$$

where both $S$ and $S_{d}$ are unit vectors. If the spacecraft rotates about the error vector $S_{e}$, then the sun vector $S$ will be driven toward the target sun vector $S_{d}$, hence the attitude error $S_{e}$ will be diminished.

\section{B. Controlled roll-yaw state}

When the sun vector gets within a certain tolerance of $S_{d}$, the sun safe mode will then transition to the controlled roll-yaw state. In this state, the main control objective is to reach and maintain a specified inclination angle between the sun vector $S$ and the spacecraft's $Z$-axis. Additionally, the spacecraft is commanded to spin about its body $Z$-axis at $\omega_{b}$ for thermal control and to spin about acquired sun vector 


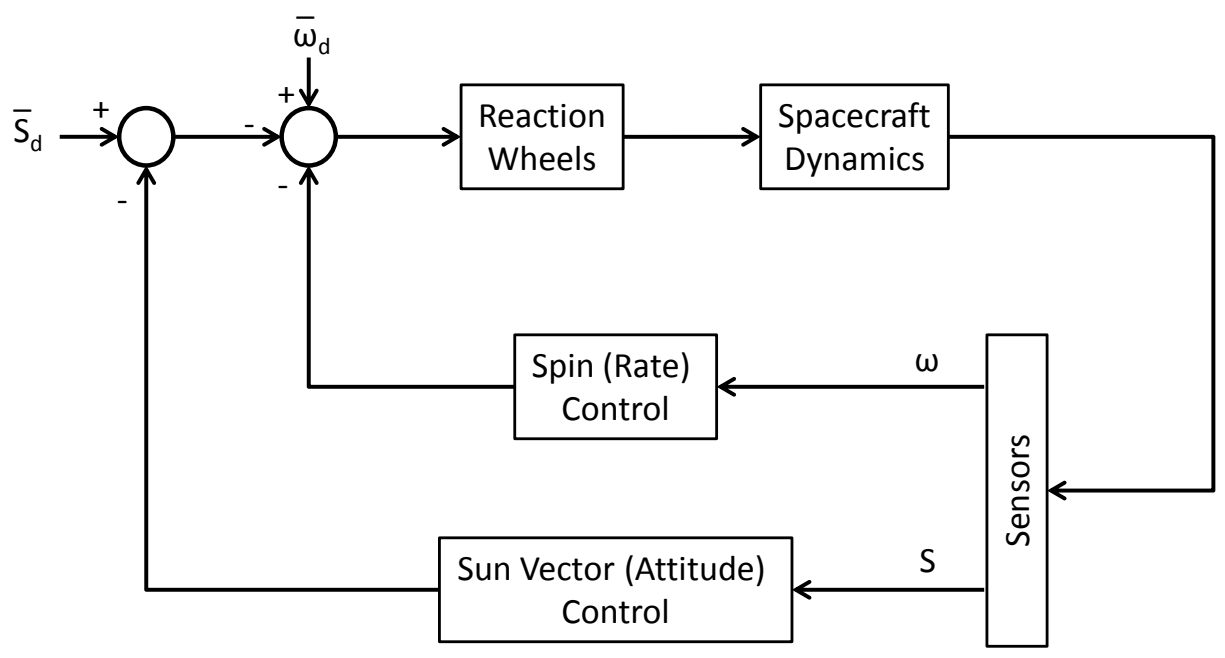

Figure 3. Schematic block diagram for initial safing state

$S$ at $\omega_{s}$ for momentum management. The $Z$-axis spin allows the heating effect from the sun to be evenly distributed, keeping all spacecraft components in the correct temperature range. The spin about $S$-axis decreases momentum build-up by allowing the lunar gravity gradient torques to act on the spacecraft in opposite directions over time. In fact, the momentum varies in a sinusoidal pattern as the spacecraft rotates.

\section{Eclipse state}

If, at any time, a minimum number of sun sensors reads zero current, then the safe mode control system asserts the eclipse flag and goes into the eclipse state. Since there is no CSS feedback at this state, the sun vector attitude loop is disabled. In order to minimize the RW control effort, the sun vector rotation command and $Z$-axis rate command become inactive. The control objective in this state is to conserve RW control authority by minimizing the interaction with the spacecraft dynamics, hence a simple rate feedback that cancels the gyroscopic effect induced by RWs is commanded. Therefore, the feedback-controlled system simply becomes the spacecraft rigid-body dynamics subject to external disturbance. Upon recovering from the eclipse state, if the spacecraft's $Z$-axis is within the required tolerance of its set point, then the safe mode returns to the controlled roll-yaw state. However, if the $Z$-axis has drifted considerably, then the safe mode returns to the initial safing state. Note however, that during the eclipse state, the spacecraft angular momentum will slowly accumulate due to the presence of external disturbance torques such as lunar gravity gradient. A momentum management solution is not considered here, however it is required to maintain controllability of the spacecraft over extended time duration. 


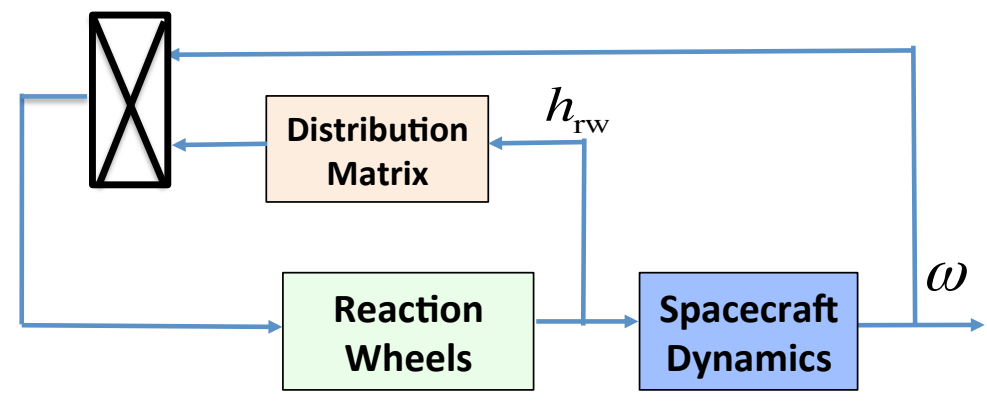

Figure 4. Schematic block diagram for eclipse state

\section{Simulation Results}

The sun safe controllers outlined in the previous sections were implemented on the LADEE spacecraft model to demonstrate that the proposed controllers can be successfully executed at each sun safe state. For this purpose, spacecraft dynamics on a science orbit around the moon were simulated. The initial spacecraft body rates were assumed to be null, which is typical of an inertially fixed science operation. The initial reaction wheels speeds were set to those of a typical science orbit momentum state for the spacecraft, while the initial sun vector was chosen to be a reasonable angle off of the $X Y$ plane. In this simulation, a desired sun angle, roll rate $\omega_{b}$ and yaw rate $\omega_{s}$ were chosen.

As shown in Figure 5, it took about 130 seconds for spacecraft to reach the desired sun elevation angle, and the body rates converged to their set-points as shown in Figure 6, with the rate about the $Z$-axis remaining constant and the $X$ and $Y$-axis rates changing to keep a constant rotation about the sun vector. The roll-yaw controller was able to maintain the spacecraft $Z$-axis tilt very close to the set point, until an eclipse was detected 2000 seconds into the orbit. As evidenced in Figure 7, the eclipse state controller was simply to null the RW's gyroscopic coupling with the spacecraft. The lunar gravity gradient torques were shown in Figure 8. As the spacecraft emerged from the eclipse at 4650 seconds, the nominal controlled roll-yaw behavior resumed as the sun vector elevation and body rates were again driven to their set points. 


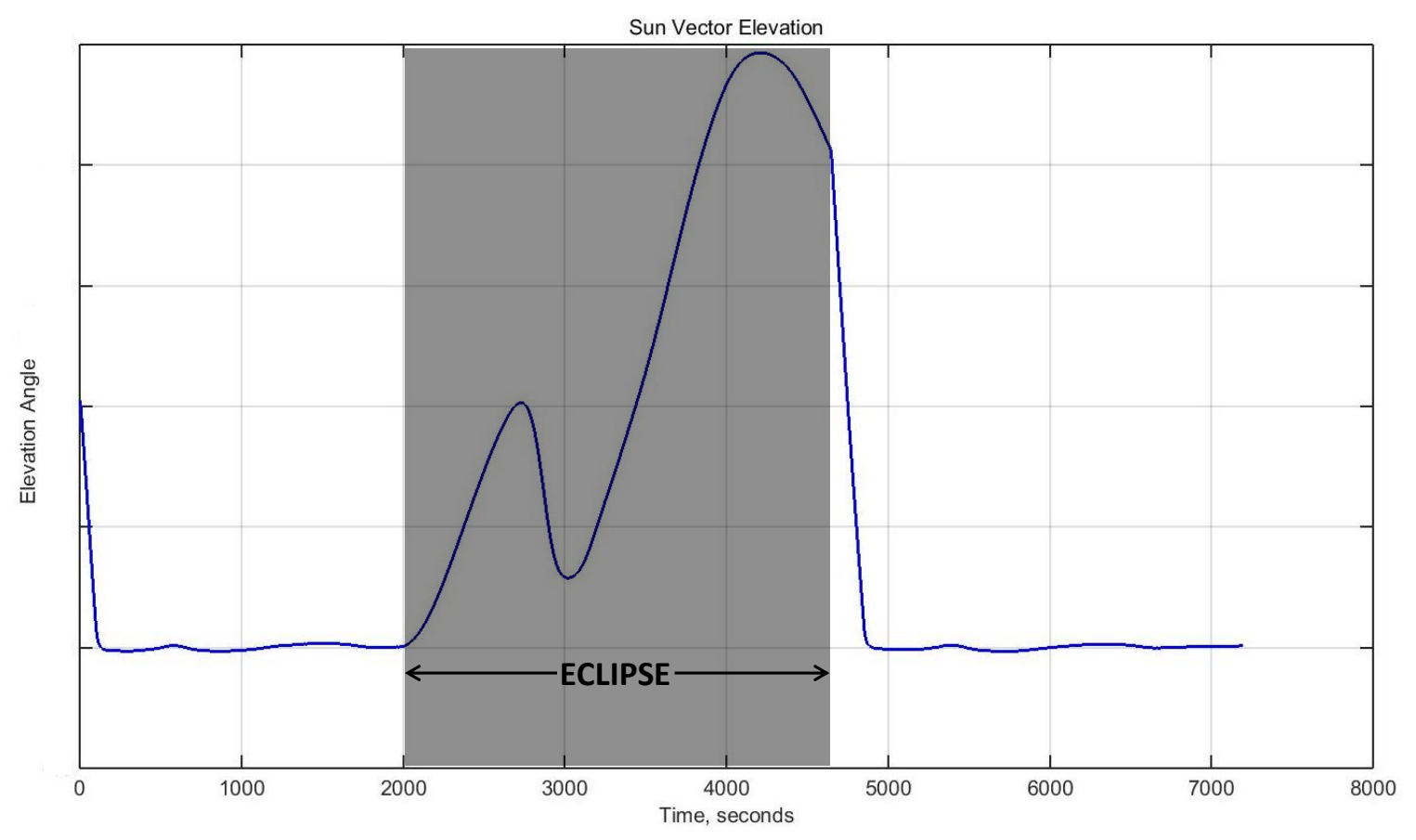

Figure 5. Simulated sun vector

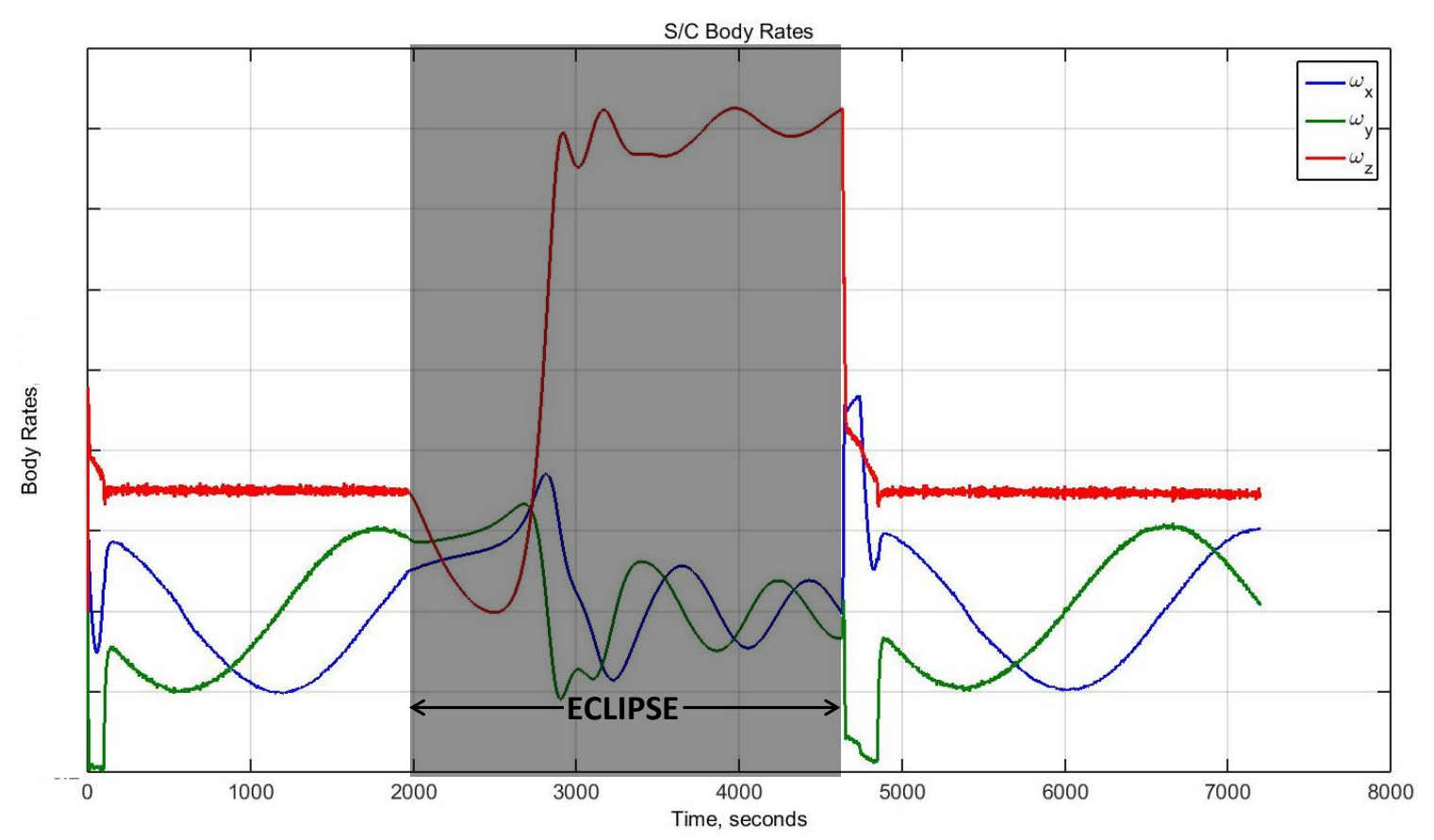

Figure 6. Simulated spacecraft body rates 


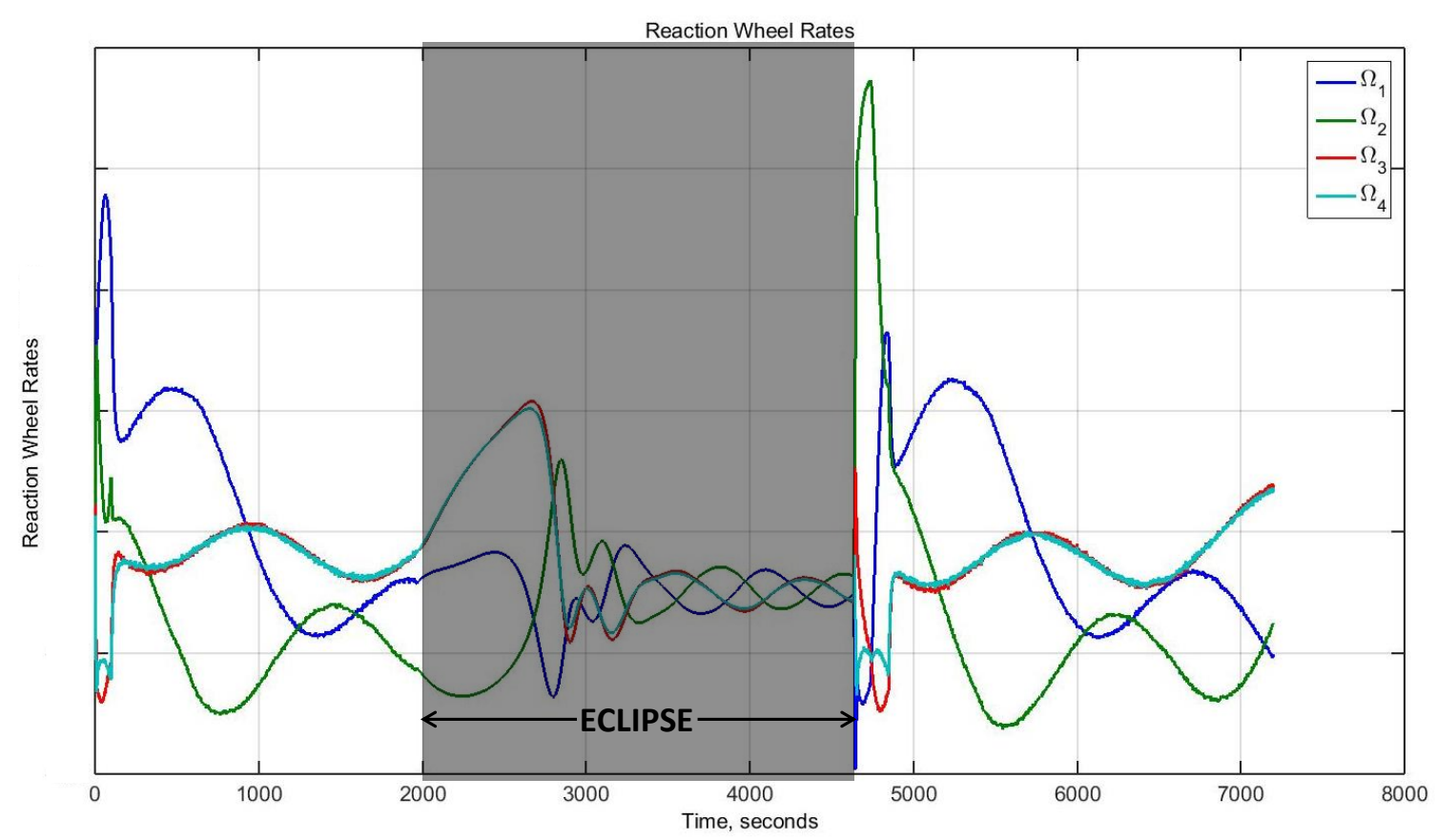

Figure 7. Simulated reaction wheel rotational rates

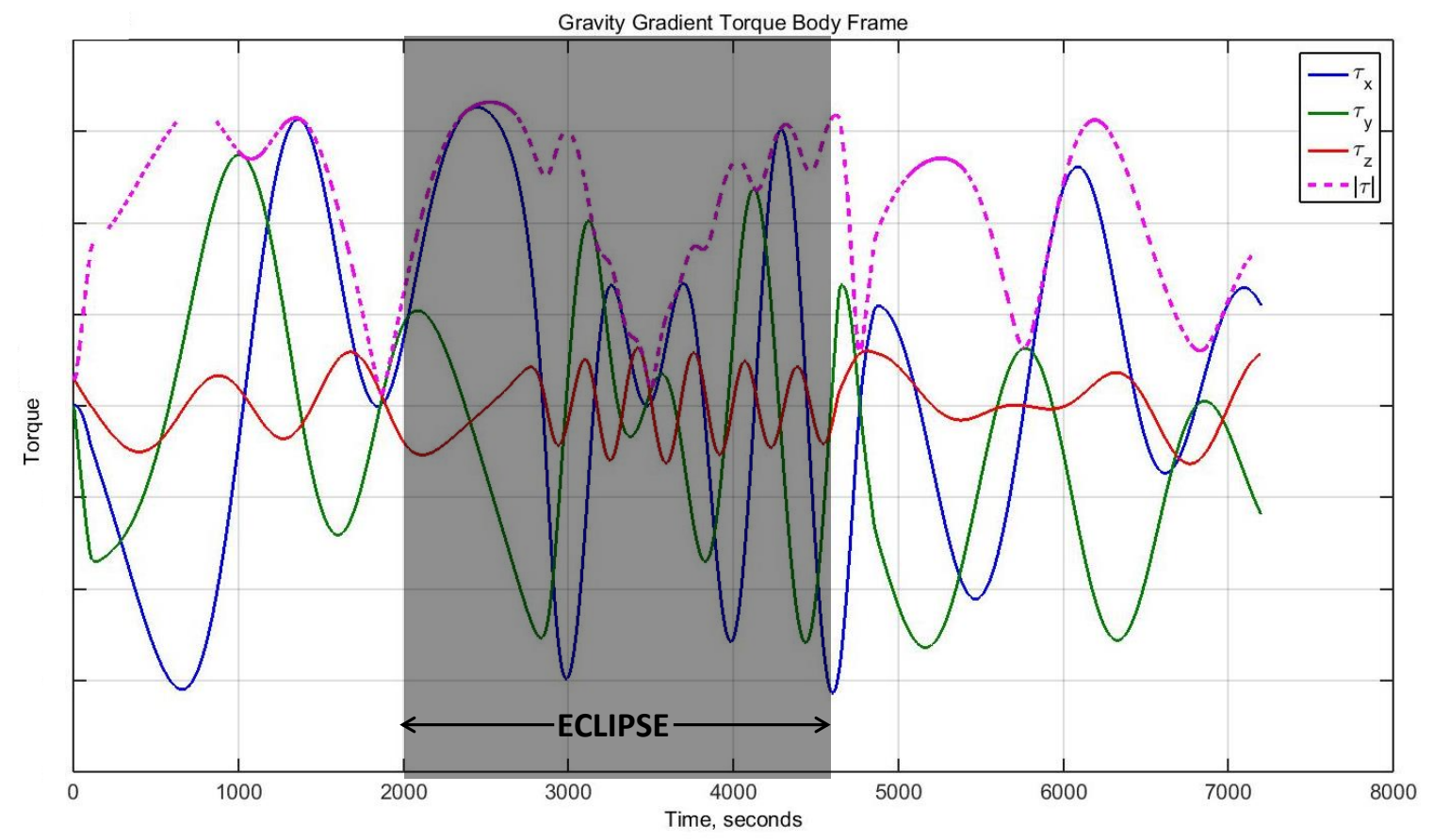

Figure 8. Simulated lunar gravity gradient torques 


\section{Orbital Flight Data}

The LADEE spacecraft was launched on a Minotaur V vehicle on September 7, 2013 UTC. Upon initial separation from the launch vehicle, the spacecraft autonomously performed a number of housekeeping tasks, then transitioned command authority to its Safe Mode (SM) Controller. Implementation of the LADEE SM control was modified slightly during the actual flight missions. The number of CSS required by the flight software to be in view of the sun, before exiting the eclipse state, was changed from three to one. The rational behind this change was that in some rare attitudes, the external hardware/instruments on the spacecraft (thrusters, payloads, etc.) could cast a shadow over a sensor, which might trigger the SM control into the eclipse state. Although using only one or two sensors resulted in a degraded estimate of sun vector, this was deemed acceptable as the spacecraft would have enough insight to orient itself into a configuration that would illuminate more sensors and hence increase accuracy. If this change had not been made, the SM control would have entered the eclipse state more frequently, and at each such event the spacecraft would have to wait for a more favorable attitude before it could resume the science missions. Another minor modification was choosing the desired sun vector to be perpendicular to the spacecraft body $Z$-axis, instead of at $+10^{\circ}$. Since the power generated from solar cells had proven to be sufficient with this new attitude, the change enabled simpler monitor and control of the sun vector dynamics. The final change in the LADEE SM control was the elimination of the initial safing state. This was a result of relaxed requirements from the payloads on-board the spacecraft. However, as demonstrated in the simulations, this control mode could still be useful for any future spacecraft.

As shown in Figure 9, the initial transition from an uncontrolled tumble to a SM controlled attitude was drastic. Within minutes, the spacecraft was able to place the sun vector at its set elevation, and begin the $Z$-axis rotation. However, looking only at the sun vector measurement would not render any insight into the rotation about the sun vector. Figure 10 depicts the spacecraft body rates from MEMS measurement, which gives a clear picture of sun vector rotation. In this figure, the achieved body rates, when the SM controller was activated, can be observed to match the commanded rates about the $Z$-axis and sun vector fairly quickly.

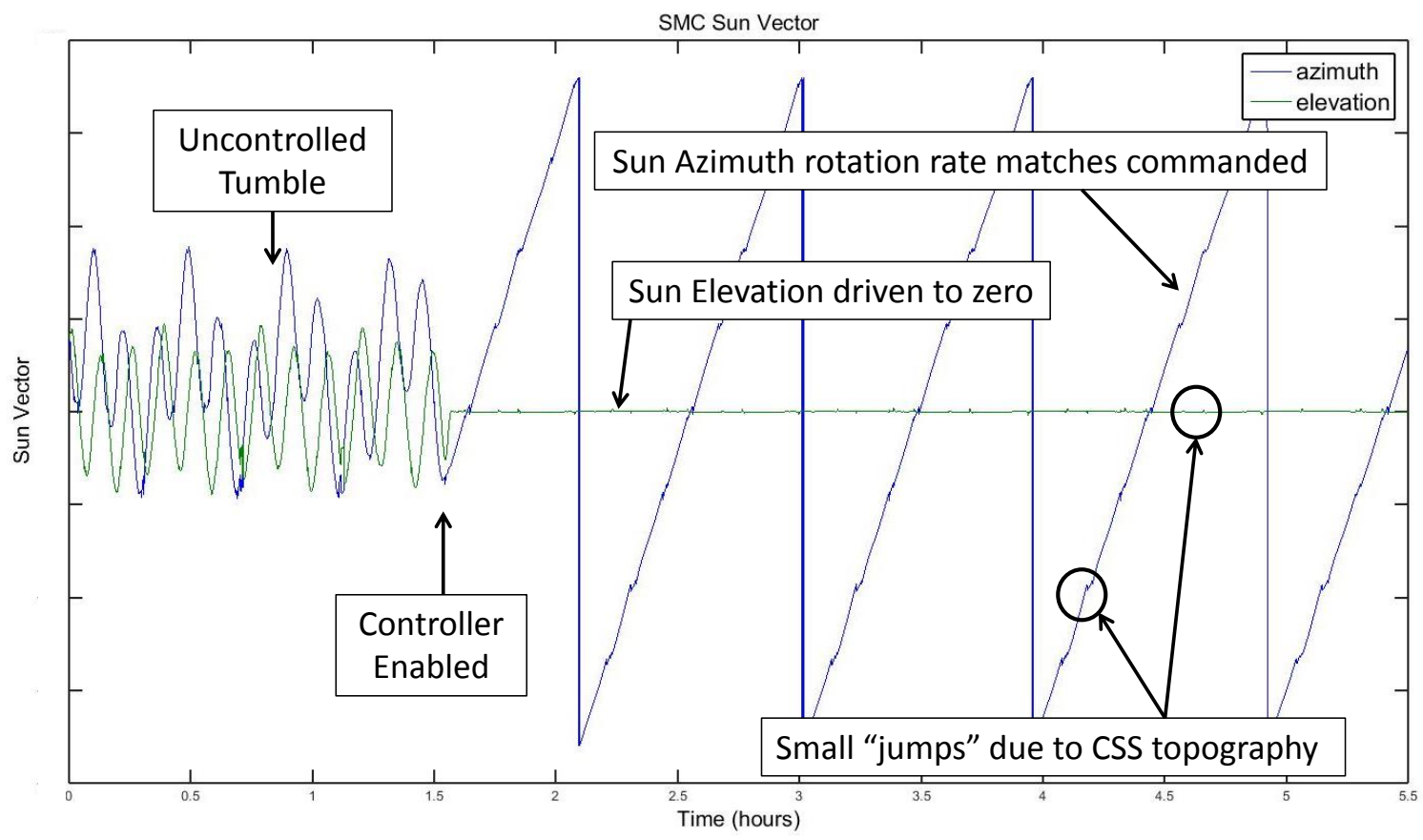

Figure 9. LADEE sun vector acquisition during startup

In addition, a close examination of Figures 9 and 10 further reveals some interesting dynamical behaviors. First, it can be seen from Figure 9 that the distributed set of 12 CSS results in small spikes or 'jumps' in the estimated sun vector. This is because, as the spacecraft rotates, the sun would enter and exit the field 


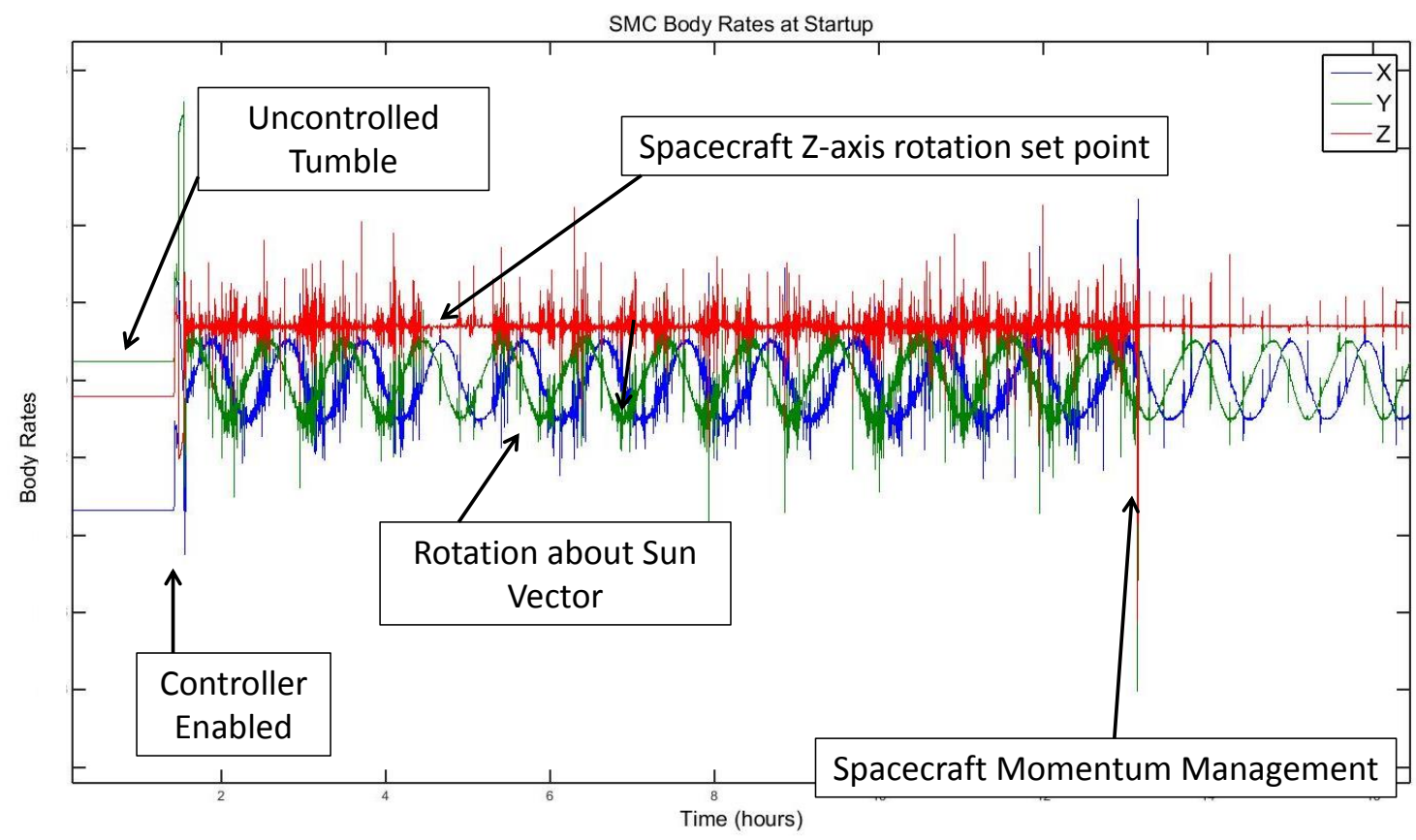

Figure 10. LADEE body rates during startup

of view of any particular CSS. Depending on the number of sensors in view, the SM control would update the estimate of sun vector relative to the spacecraft body axis. Although the effect seems small, it can be propagated through the body rate determination process, since changes in estimated attitude result in changes in reaction wheel commands, as evidenced in the small spikes in body rates shown in Figure 10. Furthermore, the effects of the reaction wheel's jitter can also be observed in the MEMS measurements. The noise level measured by the MEMS is significantly larger before the activation of momentum management (reduction). Prior to momentum reduction, the effect of the sun vector jumps was indistinguishable from sensor noise, but it became very clear afterward. The momentum reduction was accomplished by firing RCS thrusters and the maximum wheel speed was reduced by about $75 \%$.

Once the LADEE spacecraft had acquired its nominal science orbit, the sun became eclipsed by the moon roughly every two hours. This meant that in the event the spacecraft entered safe mode, it would transition from the eclipse state to the controlled roll-yaw state on a regular basis. Figures 11 and 12 show the actual flight data from one such occurrence. As can be seen, the sun vector reads all zeros during the eclipse. However, once the sun illuminates the sensors, the controller is able to promptly re-orient the spacecraft, and the spacecraft begins to rotate at the set rates.

It should be noted that, in addition to SM control, the LADEE spacecraft had a fine pointing control (FPC) system that utilized a star tracker for accurate attitude maneuver and was able to provide complete spacecraft attitude information. Therefore, as a way to validate the SM controller, the star tracker-FPC pair was used to estimate the sun vector, and the results were then compared with the estimate from the CSS-SMC pair. The two results were in good agreement, with the maximum difference less than $\pm 2^{\circ}$. This validated the accuracy of the SM control. To lower the error even further, use of fine sun-sensors (as opposed to LADEE's course sun-sensors) could be implemented.

Additional LADEE lunar orbital data confirmed that the rotation about the sun vector in safe mode helped to properly manage momentum build up. As seen in Figure 13, over the course of an orbit, the spacecraft momentum followed a small sinusoidal pattern corresponding to the rotation of the spacecraft. Overall there was a slight total momentum accumulation. However, if there had been no rotation about the sun vector, the momentum would have accumulated much faster, requiring more frequent momentum reduction operations through the firing of RCS thrusters, and therefore would have consumed excessive propellant/fuel. 


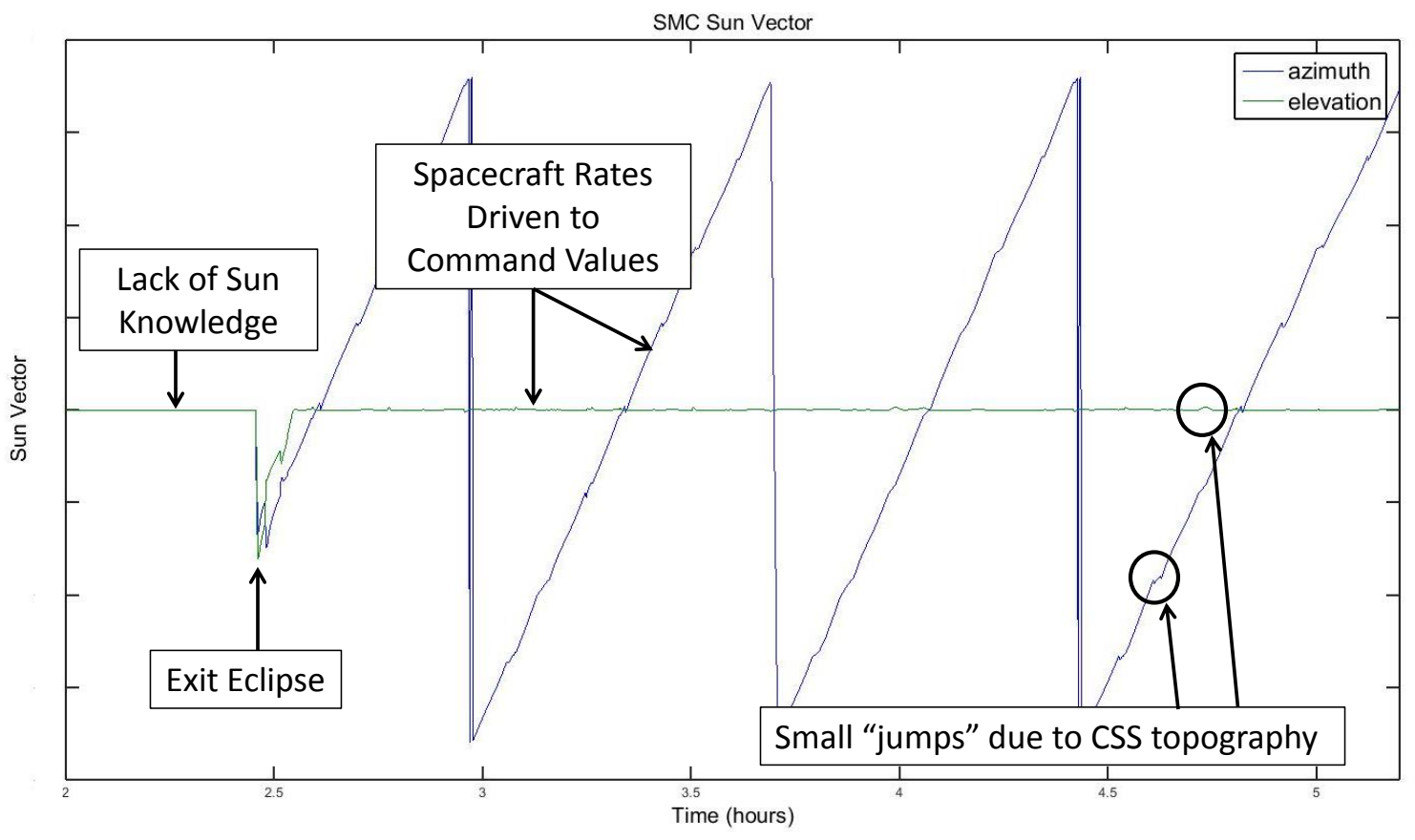

Figure 11. LADEE sun vector during eclipse exit

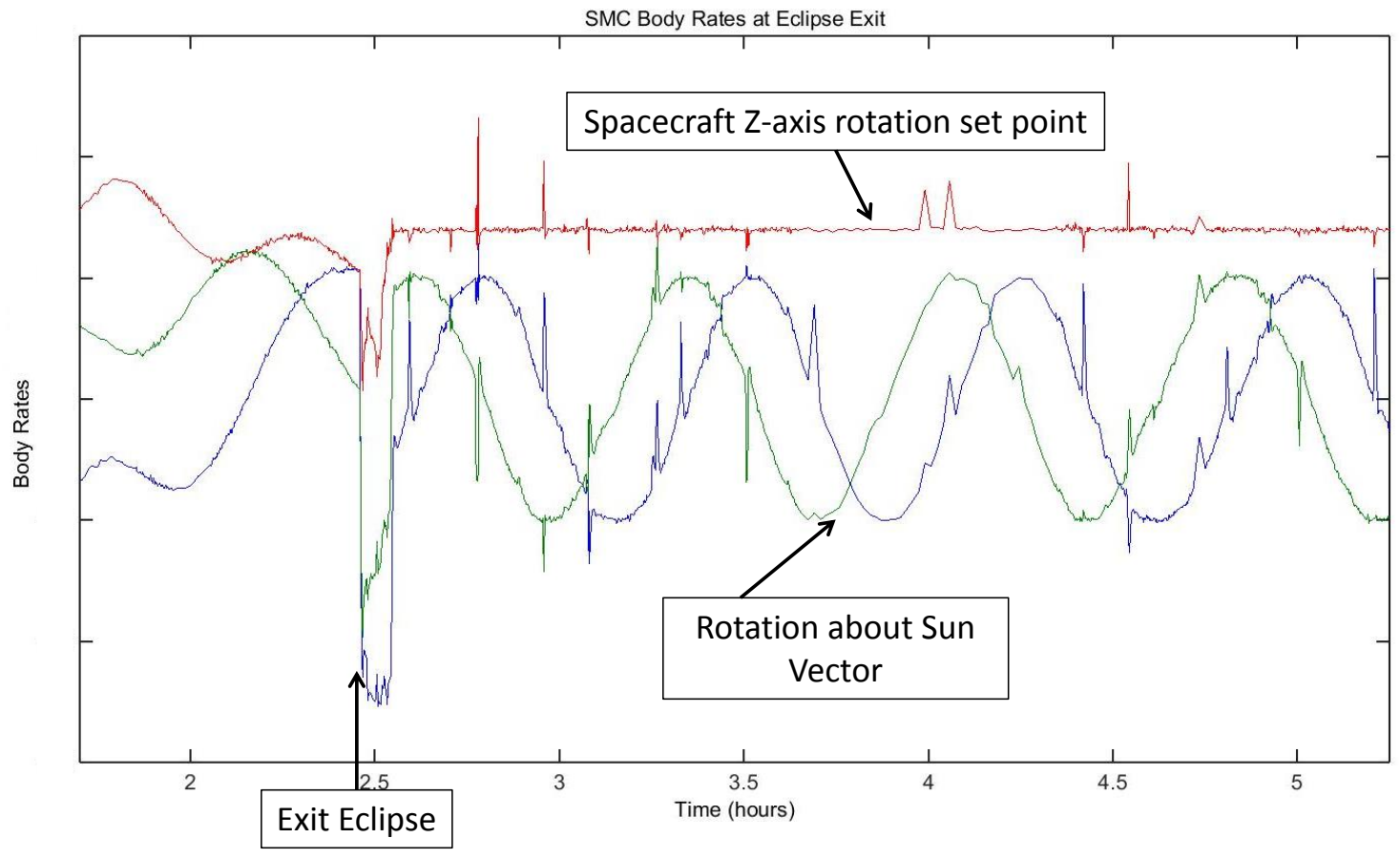

Figure 12. LADEE body rates during eclipse exit 


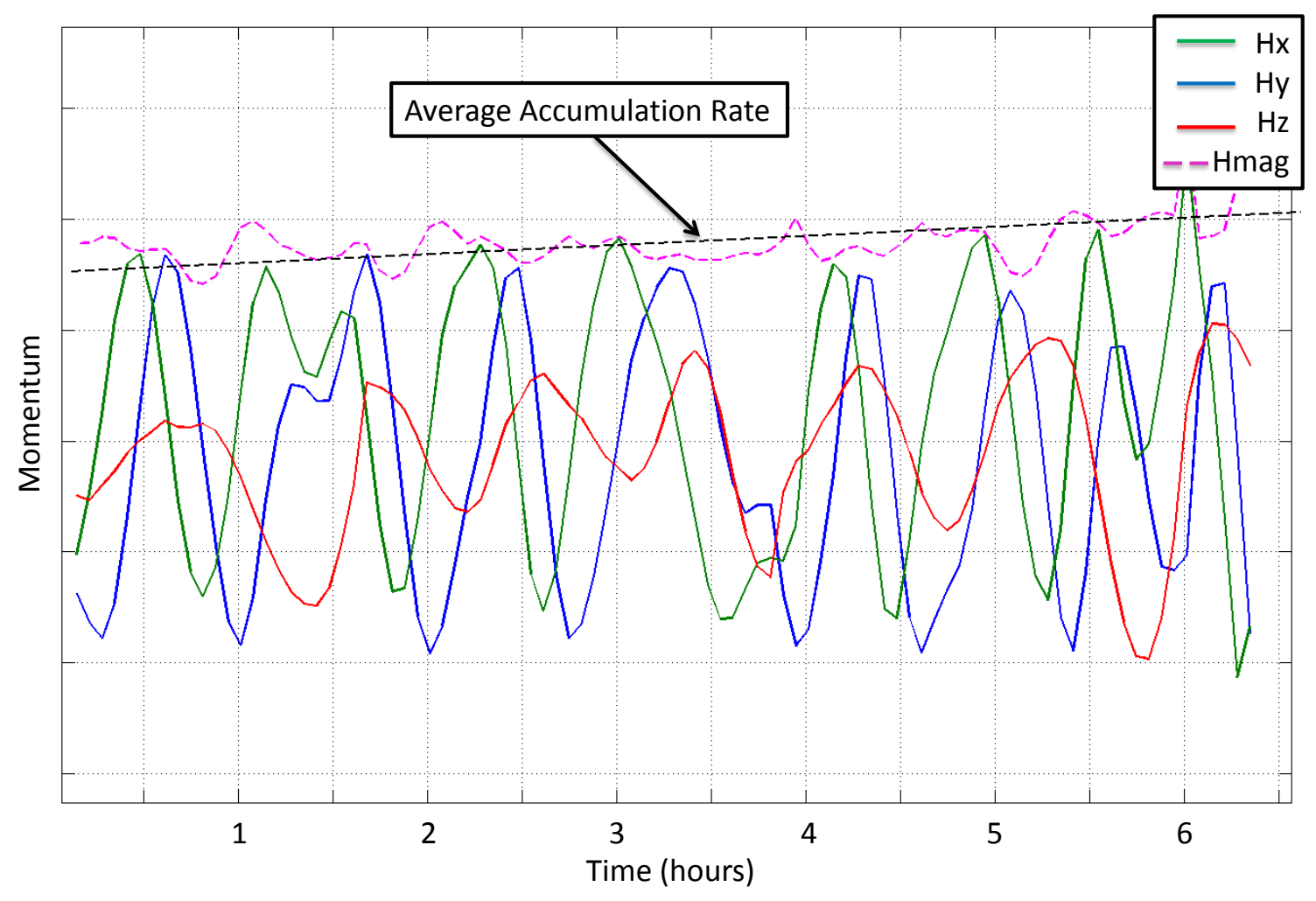

Figure 13. LADEE Momentum Accumulation during Safe Mode 


\section{Conclusions}

This paper presented the sun safe mode control design concept for LADEE. Three closely related sun safe states were defined and analyzed. The least square minimization technique was used to estimate the sun vector from CSS measured data, and the simulation results showed that this approach renders the sun vector estimation with much higher accuracy. The accuracy of sun vector tracking using CSS was further validated in actual flight through star tracker. In addition, the actual flight data indicated that during LADEE's sun safe mode the proposed SM controllers were able to position the spacecraft at a desired sun vector and maintain a specific rotational rate about the $Z$-axis as well as the sun vector.

\section{Acknowledgments}

The authors would like to thank Jeff Brown and Michael McIntyre of NASA's Ames Research Center, and Scott Starin and Paul Mason of NASA's Goddard Space Flight Center, for their support, guidance and helpful discussions. In addition, the authors would like to thank the entire LADEE team for a great experience on an amazing journey.

\section{References}

\footnotetext{
${ }^{1}$ http://nssdc.gsfc.nasa.gov/nmc/spacecraftDisplay.do?id=LADEE

${ }^{2}$ Markley, F. Landis, and John D. Nelson, "Zero-Gyro Safemode Controller for the Hubble Space Telescope." Journal of Guidance, Control, and Dynamics, Vol. 17, No. 4, 1994, pp. 815-822.

${ }^{3}$ Bourkland, Kristin L., Scott R. Starin, and David J. Mangus, "The Use of a Gyroless Wheel-Tach Controller in SDO Safehold Mode" Flight Mechanics Symposium, 2005.

${ }^{4}$ Starin, Scott R., and Kristin L. Bourkland, "Persistent Attitude Error in a Sun-Pointing Controller due to Nonlinear Dynamics," AIAA Guidance, Navigation and Control Conference, Hilton Head, SC, 2007.

${ }^{5}$ Wen, John T.Y. and K. Kreutz-Delgado, "The Attitude Control Problem," IEEE Trans. on Automatic Control, Vol. 36, No. 10, 1991, pp. 1148-1162.

${ }^{6}$ Wie, B., H. Weiss, and A. Arapostathis, "Quaternion Feedback Regulator for Spacecraft Eigenaxis Rotations," Journal of Guidance, Vol. 12, No. 3, 1989, pp. 375-380.

${ }^{7}$ Yazdi, N., F. Ayazi, and K. Najafi, "'Micromachined Inertial Sensors,"' Proceedings of the IEEE, Vol. 86, No. 8, 1998, pp. 1640-1659.

${ }^{8}$ Sidi, Marcel J., Spacecraft Dynamics and Control: A Practical Engineering Approach, Cambridge Univeristy Press, Cambridge, UK, 1997

${ }^{9}$ Swei, S., J. Fusco, and R. Nakamura, "LADEE Safe Mode Control System Analysis and Synthesis," to be submitted.

${ }^{10}$ Allgeier, Shawn E., Matt Mahin, and Norman G. Fitz-Coy, "Design and Analysis of a Coarse Sun Sensor for PicoSatellites," Proc. of AIAA Unmanned...Unlimited Conference, Seattle, WA, 2009.

${ }^{11}$ Suhas Mohite, Nishad Patil and Rudra Pratap (2006), "'Design, modelling and simulation of vibratory micromachined gyroscopes,"' International MEMS Conference 2006.
} 